\title{
HIERARCHICAL METHOD OF INTER-AREA FAST REROUTING
}

\author{
Oleksandr Lemeshko ${ }^{1}$, Oleksandra Yeremenko ${ }^{2}$, Olena Nevzorova ${ }^{3}$
}

\author{
Kharkiv National University of Radio Electronics, Department of Infocommunication Engineering \\ Kharkiv, Ukraine, 14 Nauka Ave., Ph./fax: +380577021320 \\ E-mail: 'oleksandr.lemeshko@nure.ua; ${ }^{2}$ oleksandra.yeremenko.ua@ieee.org; ${ }^{3}$ olena.nevzorova@nure.ua
}

In this paper, in order to increase the scalability and fault-tolerance of routing solutions the hierarchical method of interarea fast rerouting in communication networks was presented. The method is based on the decomposed representation of the flowbased routing model with the introduction of the area interaction conditions to ensure connectivity of the inter-area routes. The model includes conditions for border routers protection, adapted for both single path and multipath routing. During the research it was established that the efficiency of the proposed method in terms of the speed of the coordinating procedure convergence was most influenced by the number of border routers and the implemented routing strategy.

Keywords: inter-area routing, hierarchy, fault-tolerance, fast rerouting

\section{Introduction}

A distinctive feature of any complex system, to the class of which the modern communication networks also belong, is the hierarchy of its structural and functional construction. It is the hierarchical nature of the network architecture, under the conditions of constant growth in the number and nature of connections of its elements (switches, routers, servers, etc.) that maximizes the scalability of protocol solutions related to the reduction of the amount of service information circulating in the network, reduction of the sizes of routing tables stored in routers, increasing efficiency of traffic management in network. It is important to note that most modern Quality of Service (QoS) assurance tools have already been adapted to the principles of multi-level traffic management. This, above all, refers to the Hierarchical Queuing Framework, Hierarchical Policing and Hierarchical Shaping (Cisco Networking Academy, 2014).

However, the routing protocols are most sensitive to scalability problems, since their main task is to determine (calculate) the paths along which end-to-end QoS requirements would be met, which requires the coordinated operation of not one but a number of network devices. Principles of hierarchical routing are actively used in IP (Internet Protocol), ATM (Asynchronous Transfer Mode) and MPLS (Multiprotocol Label Switching) networks. This was especially evident in the functionality of such protocols as BGP (Border Gateway Protocol), OSPF (Open Shortest Path First), IS-IS (Intermediate System to Intermediate System), and PNNI (Private Network-to-Network Interface) (Cisco Networking Academy, 2014; Le Roux et al., 2005; Wright, 2003). A distinctive feature of these protocols is the division of the network into subnets of different hierarchy levels. The upper level is defined by the backbone, through which low-level subnets exchange packets, called areas, zones, or clusters. In IP networks, a three-level hierarchy can also be supported when two-level routing is implemented at the level of an autonomous system. This routing is organized, for example, using the OSPF protocol. Between autonomous systems, the routing is controlled, for example, by the BGP (Border Gateway Protocol), which determines the additional upper level of the solution hierarchy. At the same time, the boundary between subnets can pass both through routers (as in the OSPF protocol) and through the links (according to the IS-IS protocol). An example of the structural hierarchy is also the division of routers into classes: intra-area, inter-area (border), and backbone routers. However, under different protocol solutions, the names of routers may differ, as shown in Table 1 (Tiso and Teare, 2011; Wójcik et al., 2016).

Table 1. Routers classification in different protocols

\begin{tabular}{|l|l|l|l|}
\hline Protocol & Intra-area routers & Border routers & Backbone routers \\
\hline OSPF & Internal Routers (IRs) & Area Border Router (ABRs) & Backbone Routers (BRs) \\
\hline IS-IS & Routers Level 1 (L1) & Routers Level 1/Level 2 (L1/L2) & Routers Level 2 (L2) \\
\hline PNNI & Logical Nodes (LNs) & Border Nodes (BNs) & Peer Group Leaders (PGLs) \\
\hline
\end{tabular}


Furthermore, the introduced structural hierarchy of routing task solutions in modern networks is not always supported by the functional hierarchy. The Dijkstra and Bellman-Ford algorithms, which are the basis of most modern routing protocols, do not directly take into account the peculiarities of the hierarchical network construction. In some cases this is not a problem. This, for example, is typical for the scenario where each area (a subnet of the lower level) is switched to the backbone through one border router, which is the most common solution in modern networks (Tiso and Teare, 2011). In this case, both the source router and the destination router are clearly known when solving routing tasks in both the areas and the backbone. Therefore, there is no difficulty in coordinating the final solutions of the routing problems obtained separately in the areas and the backbone.

It is important to note that using a single border router for area and backbone connection significantly reduces the reliability of the network (Rak, 2015; Yeremenko et al., 2016). In the case when the border router is not able to provide its functionality, the areas connected through it become blocked with respect to the backbone and, what is very important, to other network areas. And the reasons for this situation can be:

- failure of the router caused by a breakdown;

- congestion of the border router;

- compromise of the border router in terms of network security.

Thus, in order to increase the reliability of network solutions in practice, several border routers are used more often to link separate areas and the backbone. In case of a failure in one of them, the traffic can be transmitted through the working routers. However, in the course of solving the problems of routing inter-area flows, there is a number of important features, which should be taken into account.

The first feature is related to the fact that when solving inter-area routing tasks, it is necessary to foresee the implementation of protection schemes for border routers with their failures due to the reasons given above. Thus, along with a set of primary routes, it is necessary to calculate a set of backup paths, which are bypassed with respect to the protected router.

The second feature is that there is ambiguity in the order of using border routers, which is determined, on the one hand, in the backbone, and on the other hand, in the network areas. When implementing multipath routing, this can be accompanied by a violation of connectivity of inter-area routes on the "backbone - area" boundary and under multipath routing ambiguity can also be manifested in uncoordinated traffic balancing on the border routers.

Therefore, the task of increasing the coordination of routing solutions obtained in the individual areas and the backbone is relevant. This can be achieved by revision mathematical models and methods given in the appropriate protocols of hierarchical routing. To ensure a high level of theoretical justification for proposed solutions, it is necessary to use the propositions, principles and postulates of the theory of multilevel hierarchical systems proposed by (Mesarovic et al., 2000) and developed by (Singh and Titli, 1978). This approach has already been applied in solving hierarchical traffic management problems by Lemeshko et al. $(2014,2016 a)$.

\section{Model of Hierarchical Inter-Area Fast Rerouting}

Suppose that the structure of hierarchical communication network (Fig. 1) is presented by the oriented graph $G=(M, \mathrm{E})$, where $M$ is the set of vertices modelling network routers, and $E$ is the set of graph arks modelling networks links. Let $K$ be the set of flows being circulated in a network, and $|K|=\widetilde{K}$ is the cardinality of set $K$ quantitatively characterizing the total number of flows in a network. For every $k$ th flow $\left(k \in K\right.$ ) assumed to be known its packet rate $\lambda^{k}$, which is measured in packets per second $(1 / \mathrm{s})$.

During the developing the decomposed model of inter-area routing let us suppose that the communication network consists of $n$ subnetworks or areas interconnected by principle of constructing IP networks. Then, let every separate $p$ th area in the network is described by subgraph $G^{p}=\left(M^{p}, E^{p}\right)$ of graph $G$, where $M^{p}=\left\{M_{i}^{p} ; i=\overline{1, m_{p}}\right\}$ is the set of $p$ th area routers, $m_{p}$ is the total number of routers in the area; $E^{p}=\left\{E_{i, j}^{p} ; i, j=\overline{1, m_{p}}, i \neq j\right\}$ is the set of links connecting routers of the $p$ th area $(p=\overline{0, n})$. The number 0 is assigned to the backbone area presented by the subgraph $G^{0}=\left(M^{0}, E^{0}\right)$. All other $n$ areas are exchanging packets through the backbone area. 


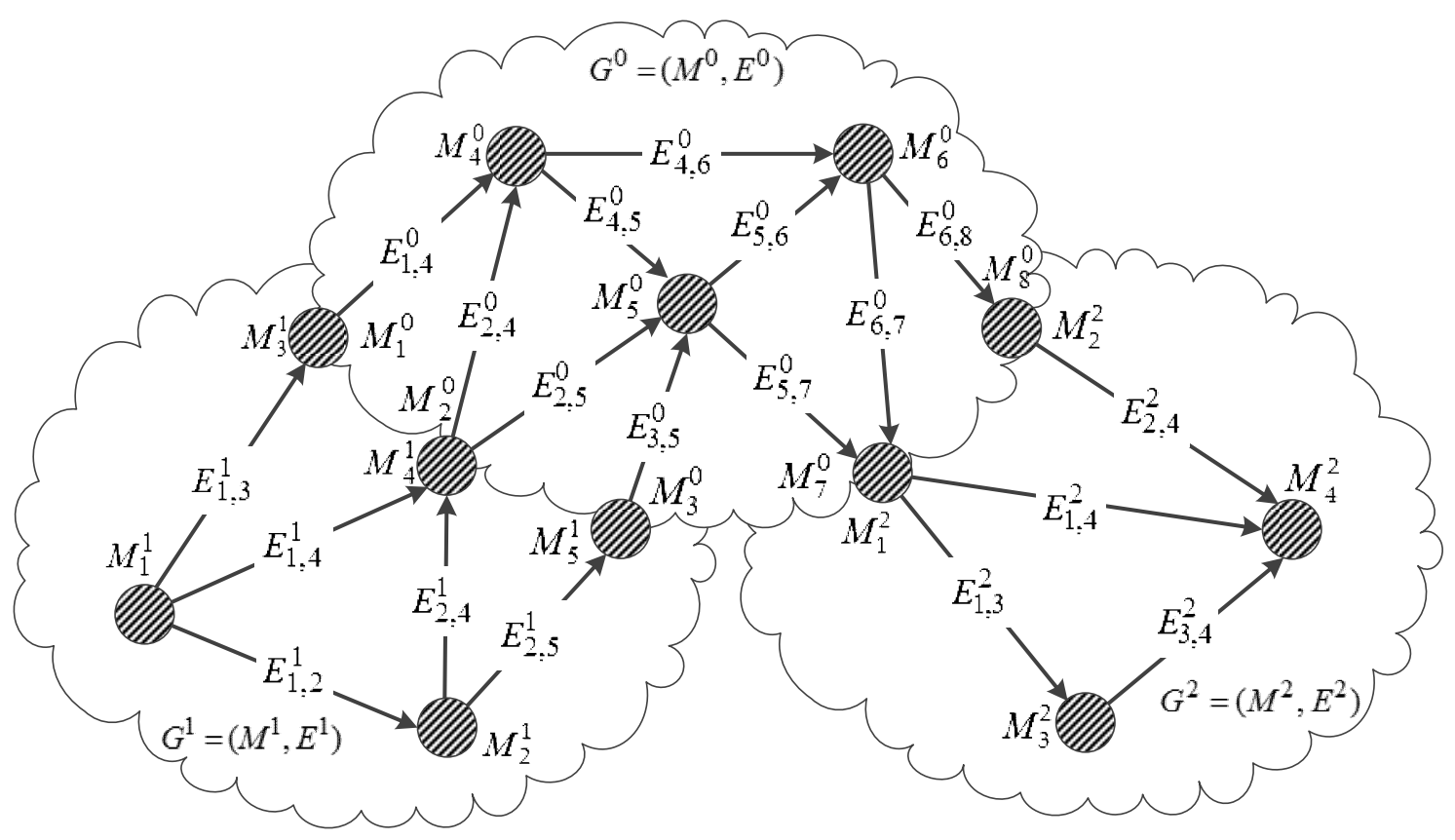

Figure 1. Example of hierarchical network

Assume that in network decomposition the boundary between areas passes through the routers like in OSPF protocol (Cisco Networking Academy, 2014). Then, based on the introduced structural network model, the following conditions occur

$$
\left\{\begin{array}{l}
M^{p} \cap M^{q}=0, \quad p, q=\overline{1, n}, \quad p \neq q ; \\
M^{0} \cap M^{p} \neq 0, \quad p=\overline{1, n} ; \\
E^{p} \cap E^{q}=0, \quad p, q=\overline{0, n}, \quad p \neq q .
\end{array}\right.
$$

Implementation of the conditions system (1) determines that only the backbone area may have the common routers with other areas. Other non-backbone areas do not have any common routers between themselves. In addition, every separate network link belongs to only one area. In order to increase the fault-tolerance of network, the number of border routers between areas has to be equal to two or more. If one of the routers fails, all flows will be redirected to other border routers.

Also for every $p$ th area let us determine the set of border routers $B^{p}\left(B^{p} \in M^{p}\right)$, through which the flows are incoming or outgoing. The whole set of the $p$ th area border routers may be split into two subsets, where $B_{i n}^{p, k}$ is the subset of border routers, through which the $k$ th flow is incoming to the $p$ th area; $B_{\text {out }}^{p, k}$ is the subset of border routers, through which the $k$ th flow is outgoing from the $p$ th area. For every link in the $p$ th area its associated bandwidth $\varphi_{(i, j)}^{p}$ is measured in packets per second (1/s). Let us denote $M_{i^{*}}^{p} \subset M^{p} \backslash B_{\text {out }}^{p, k}$ as a set of routers, which are adjacent for the router $M_{i}^{p}$.

Suppose that for the network structure shown in Fig. 1, the source of the $k$ th flow is one of the routers of the 1 st area, and the destination is the router of the 2 nd area. At the same time, the 0 th area is the backbone. Then the introduced sets of transit routers take the following form:

$$
B_{\text {out }}^{1, k}=\left\{M_{3}^{1}, M_{4}^{1}, M_{5}^{1}\right\} ; B_{\text {in }}^{0, k}=\left\{M_{1}^{0}, M_{2}^{0}, M_{3}^{0}\right\} ; B_{\text {out }}^{0, k}=\left\{M_{7}^{0}, M_{8}^{0}\right\} ; B_{\text {in }}^{2, k}=\left\{M_{1}^{2}, M_{2}^{2}\right\} .
$$

Next, we will write down the set of routers adjacent for the router $M_{4}^{1}$ of the 1 st area: $M_{4^{*}}^{1}=\left\{M_{1}^{1}, M_{2}^{1}\right\}$. The same router $M_{4}^{1}$ is designated as $M_{2}^{0}$ in the 0 th area, in which the set of adjacent routers is the following: $M_{2^{*}}^{0}=\left\{M_{4}^{0}, M_{5}^{0}\right\}$. 
Solving the problem of hierarchical routing for every $p$ th area, it is necessary to calculate routing variables $x_{(i, j)}^{p, k}$ characterizing the fraction of the $k$ th flow rate in the link $E_{i, j}^{p} \in E^{p}$. For every router in the $p$ th area, it is necessary to fulfil the condition of every $k$ th flow conservation in order to provide connectivity of calculated inter-area routes in the network (Lemeshko et al., 2016a). If the $p$ th area is the backbone area for the $k$ th flow, these conditions take the following form:

$\left\{\begin{array}{l}\sum_{B_{i n}^{0, k} \in B^{0}}\left(\sum_{E_{i, j}^{0} \in E^{0}} x_{(i, j)}^{0, k}-\sum_{E_{j, i}^{0} \in E^{0}} x_{(j, i)}^{0, k}\right)=1 ; \\ \sum_{E_{i, j}^{0} \in E^{0}} x_{(i, j)}^{0, k}-\sum_{E_{j, i}^{0} \in E^{0}} x_{(j, i)}^{0, k}=0 ; \\ \sum_{B_{o u t}^{0, k} \in B^{0}}\left(\sum_{E_{i, j}^{0} \in E^{0}} x_{(i, j)}^{0, k}-\sum_{E_{j, i}^{0} \in E^{0}} x_{(j, i)}^{0, k}\right)=-1 .\end{array}\right.$

The system of equations (2) must be performed for every packet flow separately, and the first equation in the system (2) is introduced for all backbone area routers that the $k$ th flow incomes through to the backbone area; second equation in system (2) is introduced for all backbone area routers which are the transit for the $k$ th flow in backbone area; third equation in system (2) is introduced for all backbone area routers the $k$ th flow outgoing through from the backbone area;

If the $k$ th flow was generated in the $p$ th area, and its source is, for example, the router $M_{i}^{p}$, for this network condition of every $k$ th flow conservation takes the following form:

$$
\left\{\begin{array}{l}
\sum_{E_{i, j}^{p} \in E^{p}} x_{(i, j)}^{p, k}=1 ; \\
\sum_{E_{i, j}^{p} \in E^{p}} x_{(i, j)}^{p, k}-\sum_{E_{j, i}^{p} \in E^{p}} x_{(j, i)}^{p, k}=0 ; \\
\sum_{B_{o u t}^{p, k} \in B^{p}}\left(\sum_{E_{i, j}^{p} \in E^{p}} x_{(i, j)}^{p, k}-\sum_{E_{j, i}^{p} \in E^{p}} x_{(j, i)}^{p, k}\right)=-1 .
\end{array}\right.
$$

When the router $M_{i}^{p}$ in the $p$ th area is the destination of the $k$ th flow, for this network the condition of every $k$ th flow conservation takes a following form:

$$
\left\{\begin{array}{l}
\sum_{B_{i n}^{p, k} \in B^{p}}\left(\sum_{E_{i, j}^{p} \in E^{p}} x_{(i, j)}^{p, k}-\sum_{E_{j, i}^{p} \in E^{p}} x_{(j, i)}^{p, k}\right)=1 \\
\sum_{E_{i, j}^{p} \in E^{p}} x_{(i, j)}^{p, k}-\sum_{E_{j, i}^{p} \in E^{p}} x_{(j, i)}^{p, k}=0 \\
\sum_{E_{j, i}^{p} \in E^{p}} x_{(j, i)}^{p, k}=1 .
\end{array}\right.
$$

For the case of using single path routing of flows the next conditions take place:

$x_{(i, j)}^{p, k} \in\{0,1\}$,

and for implementation of multipath routing strategy the routing variables must satisfy the conditions:

$0 \leq x_{(i, j)}^{p, k} \leq 1$. 
Routing variables (5), (6) are the coordinates of corresponding routing vectors:

$$
\vec{x}=\left[\begin{array}{c}
\vec{x}_{1} \\
\vec{x}_{2} \\
\vdots \\
\vec{x}_{p} \\
\vdots \\
\vec{x}_{N}
\end{array}\right], \vec{x}_{p}=\left[\begin{array}{c}
\vec{x}_{p}^{1} \\
\vec{x}_{p}^{2} \\
\vdots \\
\vec{x}_{p}^{k} \\
\vdots \\
\vec{x}_{p}^{\widetilde{K}}
\end{array}\right], \vec{x}_{p}^{k}=\left[\begin{array}{c}
x_{(1,2)}^{p, k} \\
x_{(1,3)}^{p, k} \\
\vdots \\
x_{(i, j)}^{p, k} \\
\vdots \\
x_{\left(m_{p}, m_{p}-1\right)}^{p, k}
\end{array}\right],
$$

where $\vec{x}$ is the vector of routing variables determining the order of flows routing in the network as a whole; the vector $\vec{x}_{p}$ determines order of flows circulated in the $p$ th area; the vector $\vec{x}_{p}^{k}$ determines the problem solution of the $k$ th flow routing in the $p$ th area.

In distributed (decentralized) calculation of the vectors $\vec{x}_{p}$ and $\vec{x}_{p}^{k}$ within each separate $p$ th area, it is important to provide inter-area connectivity of routes, i.e. of routes passing through a set of routers in different areas. Similar to the results obtained in paper (Lemeshko et al., 2016a), this implies the introduction in structure of the model (2)-(7) additional conditions of inter-area interaction:

$C_{p, 0}^{k} \vec{x}_{p}^{k}=C_{0, p}^{k} \vec{x}_{0}^{k}, p=\overline{1, n}, k \in K$,

where $C_{p, 0}^{k}$ is the matrix of interconnection between the $p$ th area and the backbone with size $m_{p, 0} \times m_{x}^{p, k} ; m_{p, 0}=\left|M^{p} \cap M^{0}\right|$ is the number of routers which are boundary between the $p$ th and backbone areas; $m_{x}^{p, k}$ is the number of coordinates $x_{(i, j)}^{p, k}$ of the vector $\vec{x}_{p}^{k}$.

\section{Conditions of Protecting Border Routers under Hierarchical Inter-Area Fast Rerouting}

When solving the problem of inter-area fast rerouting, along with the calculation of the primary routes, it is necessary to calculate a number of backup paths. Therefore, as shown in (Lemeshko et al., 2016b), additional routing variables $y_{(i, j)}^{p, k}$ are introduced. By analogy with expressions (7), the variables $y_{(i, j)}^{p, k}$ can also be represented as the coordinates of the corresponding vectors $\vec{y}, \vec{y}_{p}$ and $\vec{y}_{p}^{k}$, and conditions of inter-area interaction (8) must be met for the backup routes.

In this paper, we offer protection schemes for a border router by protecting a set of links incident to the protected node. Let one of the routers of the $p$ th area be the source of the $k$ th flow. Then, when implementing single path routing in protecting a border router $M_{i}^{p} \in B_{\text {out }}^{p, k}$, the following conditions should hold:

$y_{(j, i)}^{p, k} \in\left\{0 ; \delta_{(j, i)}^{p, k}\right\} \forall M_{j}^{p} \in M_{i^{*}}^{p}$ under $M_{i^{*}}^{p} \subset M^{p} \backslash B_{o u t}^{p, k}$,

where

$\delta_{(j, i)}^{p, k}=\left\{\begin{array}{l}0, \text { when protecting the link } E_{j, i}^{p} \\ 1, \quad \text { otherwise. }\end{array}\right.$

In case of multipath routing the following conditions should be fulfilled:

$0 \leq y_{(j, i)}^{p, k} \leq \delta_{(j, i)}^{p, k} \forall M_{j}^{p} \in M_{i^{*}}^{p}$ under $M_{i^{*}}^{p} \subset M^{p} \backslash B_{\text {out }}^{p, k}$,

where the selection of values $\delta_{(j, i)}^{p, k}$ obeys the condition (10). 
In notations of the $\quad 0$ th area (backbone) for the router being protected $M_{z}^{0} \in B_{i n}^{0, k}$ the same conditions are written as follows:

$y_{(z, j)}^{0, k} \in\left\{0 ; \delta_{(z, j)}^{0, k}\right\} \forall M_{j}^{0} \in M_{z^{*}}^{0}$ under $M_{z^{*}}^{0} \subset M^{0} \backslash B_{i n}^{0, k}$.

When implementing the strategy of multipath routing, we have the conditions:

$0 \leq y_{(z, j)}^{0, k} \leq \delta_{(z, j)}^{0, k} \forall M_{j}^{0} \in M_{z^{*}}^{0}$ under $M_{z^{*}}^{0} \subset M^{0} \backslash B_{i n}^{0, k}$.

If one of the routers of the $p$ th area is the destination of the $k$ th flow, then when implementing the single path routing strategy during the protection of the border router $M_{i}^{p} \in B_{i n}^{p, k}$, the following conditions must be met:

$y_{(i, j)}^{p, k} \in\left\{0 ; \delta_{(i, j)}^{p, k}\right\} \forall M_{j}^{p} \in M_{i^{*}}^{p}$ under $M_{i^{*}}^{p} \subset M^{p} \backslash B_{i n}^{p, k}$,

where

$\delta_{(i, j)}^{p, k}=\left\{\begin{array}{l}0, \text { when protecting the link } E_{i, j}^{p} \\ 1, \quad \text { otherwise. }\end{array}\right.$

In case of multipath routing we have the following conditions:

$0 \leq y_{(i, j)}^{p, k} \leq \delta_{(i, j)}^{p, k} \forall M_{j}^{p} \in M_{i^{*}}^{p}$ under $M_{i^{*}}^{p} \subset M^{p} \backslash B_{i n}^{p, k}$,

where the selection of values $\delta_{(i, j)}^{p, k}$ obeys the condition (15).

In notations of the 0 th area (backbone) for the router being protected $M_{z}^{0} \in B_{\text {out }}^{0, k}$ these conditions are written as follows:

$y_{(j, z)}^{0, k} \in\left\{0 ; \delta_{(j, z)}^{0, k}\right\} \forall M_{j}^{0} \in M_{z^{*}}^{0}$ under $M_{z^{*}}^{0} \subset M^{0} \backslash B_{\text {out }}^{0, k}$.

When implementing the strategy of multipath routing, we have the conditions:

$0 \leq y_{(j, z)}^{0, k} \leq \delta_{(j, z)}^{0, k} \forall M_{j}^{0} \in M_{z^{*}}^{0}$ under $M_{z^{*}}^{0} \subset M^{0} \backslash B_{\text {out }}^{0, k}$.

If it is necessary to perform reservation of network bandwidth, and also to prevent possible congestion in the implementation of both single path and multipath routing strategies, the following conditions are introduced into the model, similar to the results obtained in (Lemeshko et al., 2016b):

$\frac{1}{2} \sum_{k \in K}\left(\lambda^{k}\left[\left(x_{(i, j)}^{p, k}+y_{(i, j)}^{p, k}\right)+\sqrt{\left(x_{(i, j)}^{p, k}-y_{(i, j)}^{p, k}\right)^{2}}\right]\right) \leq \varphi_{(i, j)}^{p}$.

\section{Hierarchical Method of Inter-Area Fast Rerouting}

Given the mathematical model (2)-(7) proposed above, the basis of the proposed method of hierarchical routing is the solving of optimization problem during calculation of routing variable vectors $\vec{x}_{p}^{k}(p=\overline{0, n}, k \in K)$ with fulfilment of limitations (2)-(6), (8) during the usage of the following optimality criterion:

$\min _{x, y} F, F=\sum_{p=0}^{n} \sum_{k \in K}\left[\left(\vec{x}_{p}^{k}\right)^{t} H_{p}^{k} \vec{x}_{p}^{k}+\left(\vec{y}_{p}^{k}\right)^{t} H_{p}^{k} \vec{y}_{p}^{k}\right]$,

where $H_{p}^{k}$ is diagonal matrix of weight coefficients, the coordinates of which are generally routing metrics of the $p$ th area links, $[\cdot]^{t}$ is transpose function of the vector (matrix). Objective function $F$ 
numerically characterizes total conditional cost for the organization of inter-area routing process in communication network.

Since the calculation of routes in different areas is performed independently from each other, the inter-area route connectivity is not generally ensured, i.e. the conditions of inter-area interaction (8) is not fulfilled. The initial choice of routes in each of the areas is dictated by the fact that they are optimal, i.e. the "shortest" in the framework of selected metrics $H_{p}^{k}$. As a rule, these routes are, on the one hand, the most productive; on the other hand, they contain the minimum number of hops because the additive criterion is used. The coordination, which is carried out on the upper level of hierarchy of routing solutions, obtained from separate areas, should be directed to the fulfilment of the conditions of inter-area interaction (8). To do this, during solving the formulated optimization problem involving with minimization of expression (20) with constraints (2)-(6) to provide fulfilment of interaction conditions (8), the goal coordination principle (Mesarovic et al., 2000; Singh and Titli, 1978) has been used. Then, turning to the unconditional extremum problem:

$$
\min _{x, y} F=\max _{\mu, \eta} L
$$

it is necessary to maximize following Lagrangian by $\vec{\mu}$ and $\vec{\eta}$ :

$$
\begin{aligned}
& L=\sum_{p=1}^{N} \sum_{k \in K}\left[\left(\vec{x}_{p}^{k}\right)^{t} H_{p}^{k} \vec{x}_{p}^{k}+\left(\vec{y}_{p}^{k}\right)^{t} H_{p}^{k} \vec{y}_{p}^{k}\right]+ \\
& +\sum_{p=1}^{N} \sum_{k \in K} \vec{\mu}_{p, 0}^{k}\left(C_{p, 0}^{k} \vec{x}_{p}^{k}-C_{0, p}^{k} \vec{x}_{0}^{k}\right)+\sum_{p=1}^{N} \sum_{k \in K} \vec{\eta}_{p, 0}^{k}\left(C_{p, 0}^{k} \vec{y}_{p}^{k}-C_{0, p}^{k} \vec{y}_{0}^{k}\right),
\end{aligned}
$$

where $\vec{\mu}$ and $\vec{\eta}$ are the vectors of Lagrange multipliers; $\vec{\mu}_{p, 0}^{k}$ and $\vec{\eta}_{p, 0}^{k}$ are subvectors of vectors $\vec{\mu}$ and $\vec{\eta}$ assigned to each of the vector-matrix conditions of inter-area interaction (8) for primary and backup routes respectively.

Under the goal coordination principle, the vectors of Lagrange multipliers $\vec{\mu}$ are calculated on the upper level. These multipliers are known values for lower level, that is why the expression (21) can be represented in the following decomposed form:

$$
\begin{aligned}
& L=\sum_{p=0}^{n} L_{p}, \\
& L_{0}=\sum_{k \in K}\left[\left(\vec{x}_{0}^{k}\right)^{t} H_{0}^{k} \vec{x}_{0}^{k}+\left(\vec{y}_{0}^{k}\right)^{t} H_{0}^{k} \vec{y}_{0}^{k}\right] \\
& L_{p}=\sum_{k \in K}\left[\left(\vec{x}_{p}^{k}\right)^{t} H_{p}^{k} \vec{x}_{p}^{k}+\left(\vec{y}_{p}^{k}\right)^{t} H_{p}^{k} \vec{y}_{p}^{k}\right]+\sum_{k \in K_{p}^{+}} \vec{\mu}_{p, 0}^{k} C_{p, 0}^{k} \vec{x}_{p}^{k}-\sum_{k \in K_{p}^{-}} \vec{\mu}_{q, 0}^{k} C_{p, 0}^{k} \vec{x}_{p}^{k}+ \\
& +\sum_{k \in K_{p}^{+}} \vec{\eta}_{p, 0}^{k} C_{p, 0}^{k} \vec{y}_{p}^{k}-\sum_{k \in K_{p}^{-}} \vec{\eta}_{q, 0}^{k} C_{p, 0}^{k} \vec{y}_{p}^{k}, p=\overline{1, n},
\end{aligned}
$$

where $K_{p}^{+}$is the subset of flows incoming from other network areas to the $p$ th area, which is the destination area for the $k$ th flow; $K_{p}^{-}$is the subset of flows outgoing from the $p$ th area, which is the source area for the $k$ th flow $\left(K_{p}^{+}, K_{p}^{-} \in K\right)$.

According to the functional decomposition in expression (22), all terms are attributed to the $p$ th area, wherein the second, third, fourth, and fifth terms in this expression are responsible for the coordination of routing order for primary and backup routes of outgoing and incoming flows respectively for $p$ th area in the network.

Thus, the total problem of inter-area routing was formulated as a problem of a hierarchical coordination of two-level optimization. On the lower hierarchical level there is a calculation of routing 
variables presented by vectors $\vec{x}_{p}^{k}(p=\overline{0, n}, k \in K)$. The main aim of the upper level in the proposed method is a coordination of the solutions obtained from the lower level, in order to ensure conditions of inter-area interworking (8) by modifying vectors of Lagrange multipliers in the implementation of the gradient iterative procedure (Singh and Titli, 1978):

$\vec{\mu}_{p, 0}^{k}(\alpha+1)=\vec{\mu}_{p, 0}^{k}(\alpha)+\nabla \vec{\mu}_{p, 0}^{k} ; \quad \vec{\eta}_{p, 0}^{k}(\alpha+1)=\vec{\eta}_{p, 0}^{k}(\alpha)+\nabla \vec{\eta}_{p, 0}^{k}$

where $\alpha$ is the iteration number; $\nabla \vec{\mu}_{p, 0}^{k}$ and $\nabla \vec{\eta}_{p, 0}^{k}$ are the gradients of function (22) calculated according to solutions of routing tasks $\vec{x}_{p}^{k^{*}}$ and $\vec{y}_{p}^{k^{*}}(p=\overline{0, n}, k \in K)$ received from the lower level in each separate area:

$\left.\nabla \vec{\mu}_{p, 0}^{k}(x)\right|_{x=x^{*}}=C_{p, 0}^{k} \vec{x}_{p}^{k}-C_{0, p}^{k} \vec{x}_{0}^{k} ;\left.\quad \nabla \vec{\eta}_{p, 0}^{k}(y)\right|_{y=y^{*}}=C_{p, 0}^{k} \vec{y}_{p}^{k}-C_{0, p}^{k} \vec{y}_{0}^{k}, \quad p \neq 0$.

On approaching the values of gradient (24) coordinates to zero the connectivity of inter-area routes will be provided. The effectiveness of the proposed method of inter-area fast rerouting in terms of efficiency and optimality of the obtained solutions depends on the coordinating procedure convergence rate (23)-(24). Technologically the lesser number of iterations is needed to obtain optimal solution, the lesser volume of overhead transmitted between hierarchical levels about the results of calculations on each of the iterations in the network is, and the lesser time of solving the routing problem in communication network as a whole is.

\section{Numerical Study and Analysis of Hierarchical Method of Inter-Area Fast Rerouting}

\subsection{Description of Initial Data}

Let us check the efficiency of the proposed method with the confirmation of the adequacy of the mathematical model assumed in its basis on a certain numerical example. When investigating the solution of the problem of hierarchical inter-area fast rerouting within the framework of this example, the structure of the communication network, shown in Fig. 2, has been chosen. This network consists of three areas: Area 1, Area 2, and backbone Area 0.

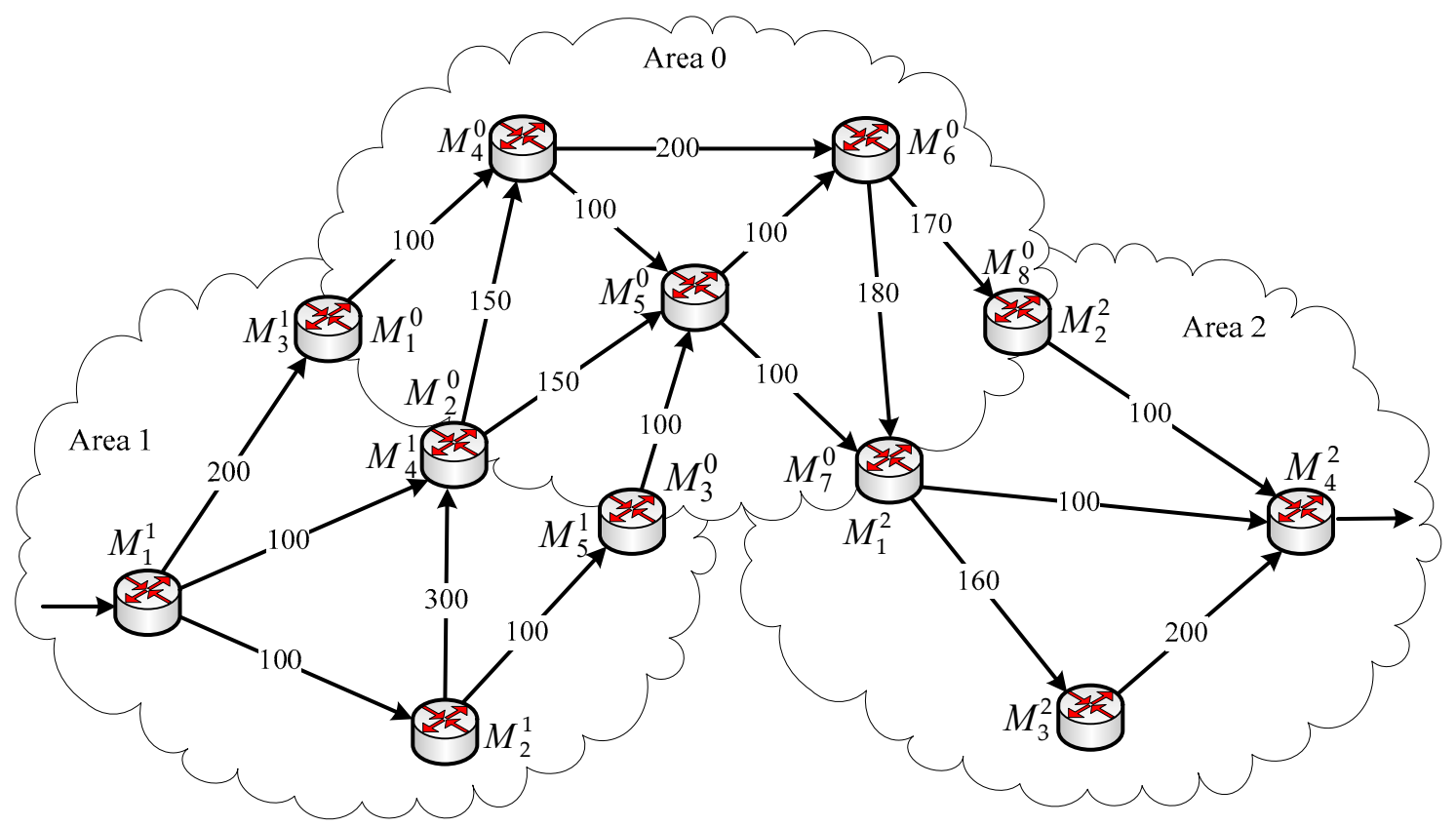

Figure 2. The structure of the network being studied 
Routers that are in the Area 1, Area 2 and Area 0, respectively, can be conveniently represented in the form of the following sets:

- $M^{1}=\left\{M_{1}^{1}, M_{2}^{1}, M_{3}^{1}, M_{4}^{1}, M_{5}^{1}\right\}$;

- $M^{2}=\left\{M_{1}^{2}, M_{2}^{2}, M_{3}^{2}, M_{4}^{2}\right\}$;

- $M^{0}=\left\{M_{1}^{0}, M_{2}^{0}, M_{3}^{0}, M_{4}^{0}, M_{5}^{0}, M_{6}^{0}, M_{7}^{0}, M_{8}^{0}\right\}$.

The interaction between the first area and the backbone area is carried out through the routers $M_{3}^{1}$ $\left(M_{1}^{0}\right), M_{4}^{1}\left(M_{2}^{0}\right)$ and $M_{5}^{1}\left(M_{3}^{0}\right)$. In turn, the interaction between the backbone area and the second area is performed through the routers $M_{7}^{0}\left(M_{1}^{2}\right)$ and $M_{8}^{0}\left(M_{2}^{2}\right)$. Suppose that the source node sending the packets is the router $M_{1}^{1}$ located in the first area, and the destination node is the router $M_{4}^{2}$ located in the second area. Fig. 2 shows their bandwidth (1/s) in the gaps of the links. Then, by analogy with the values of the routing metrics that are specific to the OSPF protocol, the expressions $10^{8} / \varphi_{(i, j)}^{p}$ located on the main diagonal of the matrix $H_{p}^{k}$ act as the link metric. In the course of the numerical study, the intensity of the transmitted packet flow during the implementation of the single path routing strategy varied from 10 to 100 1/s, and under multipath routing - from 10 to 200 1/s.

\subsection{Research of Single Path Hierarchical Inter-Area Fast Rerouting}

Suppose that in the network, the structure of which is shown in Fig. 2, it is necessary to protect the border router $M_{4}^{1}\left(M_{2}^{0}\right)$ located on the boundary of the first and the backbone areas. With a flow rate ranging from 10 to $100 \mathrm{l} / \mathrm{s}$, the single path routing has been implemented. The resulting solution to the problem of inter-area fast rerouting represented by the primary route is shown in Fig. 3, and by the backup one - in Fig. 4. In this case, the calculated (primary or backup) path is represented by a solid line, while unused links are shown by semitransparent lines. In Fig. 3 and Fig. 4, in the gaps of the links, a fraction is indicated, where the numerator represents the intensity of the transmitted packet flow, and the denominator represents the capacity of the given link.

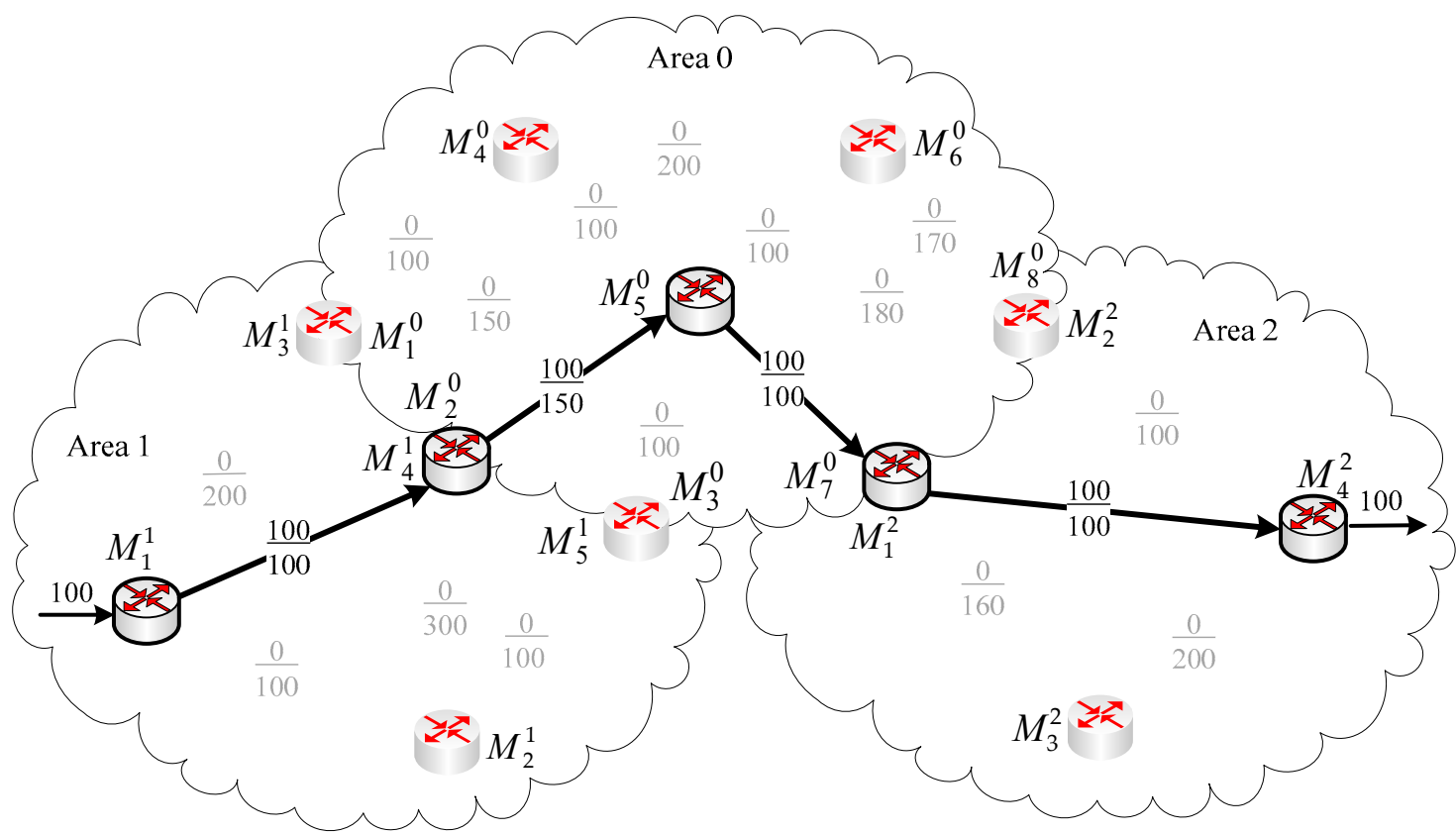

Figure 3. The resulting (coordinated) solution for the problem of inter-area single path routing of the flow with the intensity of $100 \mathrm{1} / \mathrm{s}$, represented by the primary route 
The calculated primary path, shown in Fig. 3, includes the following routers of different areas, including the border router $M_{4}^{1}\left(M_{2}^{0}\right)$ :

$M_{1}^{1} \rightarrow M_{4}^{1}\left(M_{2}^{0}\right) \rightarrow M_{5}^{0} \rightarrow M_{7}^{0}\left(M_{1}^{2}\right) \rightarrow M_{4}^{2}$

In turn, the backup route is shown in Fig. 4:

$$
M_{1}^{1} \rightarrow M_{2}^{1} \rightarrow M_{5}^{1}\left(M_{3}^{0}\right) \rightarrow M_{5}^{0} \rightarrow M_{7}^{0}\left(M_{1}^{2}\right) \rightarrow M_{4}^{2}
$$

and does not contain the protected border router:

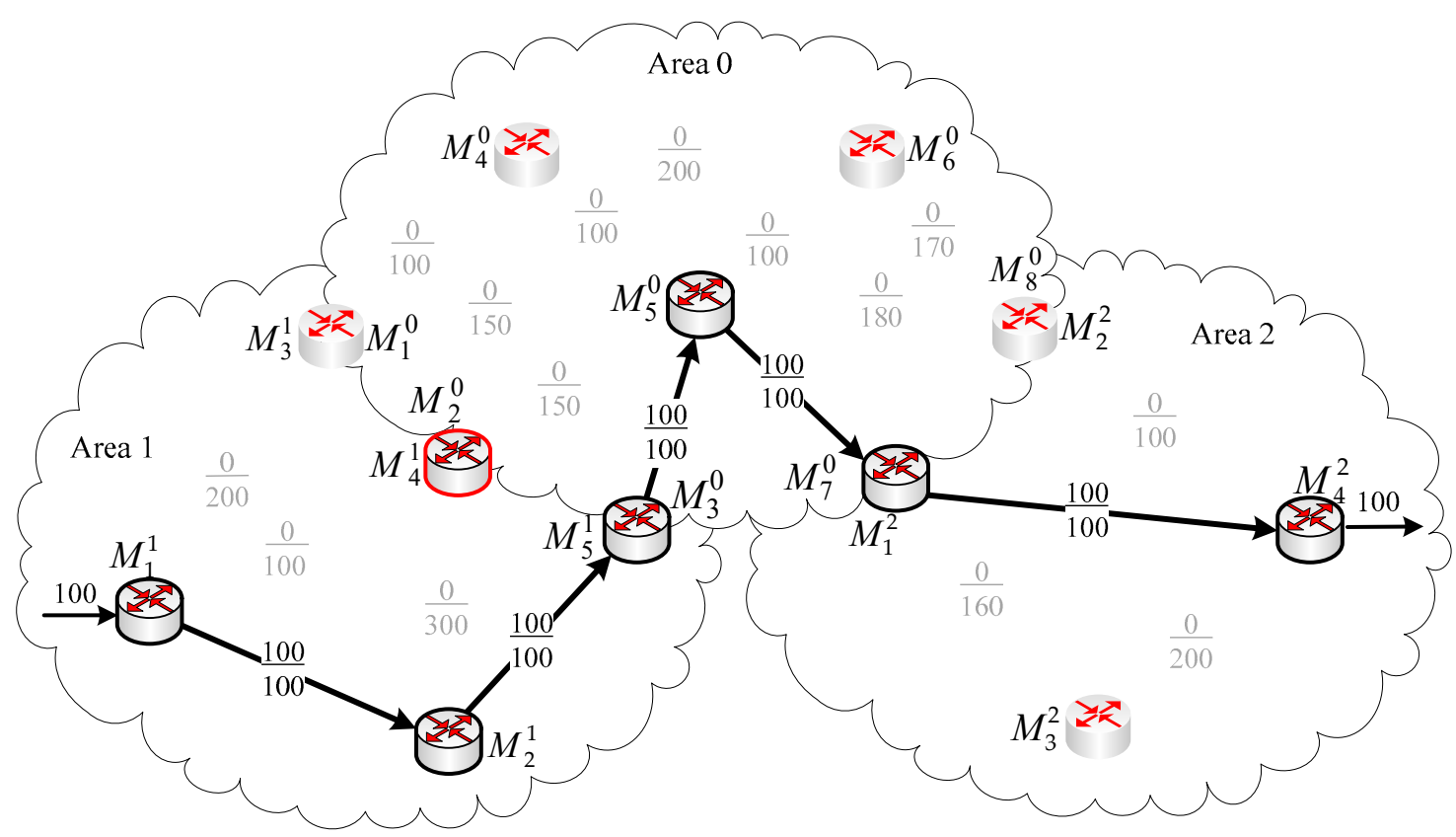

Figure 4. The resulting (coordinated) solution for the problem of inter-area single path routing of the flow with the intensity of $1001 / \mathrm{s}$, represented by the backup route

Each of the calculated routes, in accordance with condition (19), provides the transmitted flow with the necessary bandwidth. This solution was obtained after the second coordinating iteration (23), (24), since during the initial calculation the connectivity of the inter-area route was not provided, because the inter-area interaction condition (8) was not satisfied.

\subsection{Research of Multipath Hierarchical Inter-Area Fast Rerouting}

With the further increase in the intensity of the same flow up to $2001 / \mathrm{s}$ for the same initial data on the network structure and bandwidth of the links (Fig. 2), the convergence of the coordinating procedure has been already ensured after the third iteration, and the route solutions have been of the multipath nature. The notations in the gaps of the links are identical to those shown in Fig. 3 and Fig. 4. Each of the areas implements the multipath routing, because none of the links can cope with such a load. Prior to the coordination of route solutions received in the individual areas, connectivity of inter-area routes has not been provided.

As before, it is needed to protect the border router $M_{4}^{1}\left(M_{2}^{0}\right)$, and the resulting solution to the multipath inter-area fast rerouting problem is shown in Fig. 5 and Fig. 6 for the primary and the backup routes, respectively. 


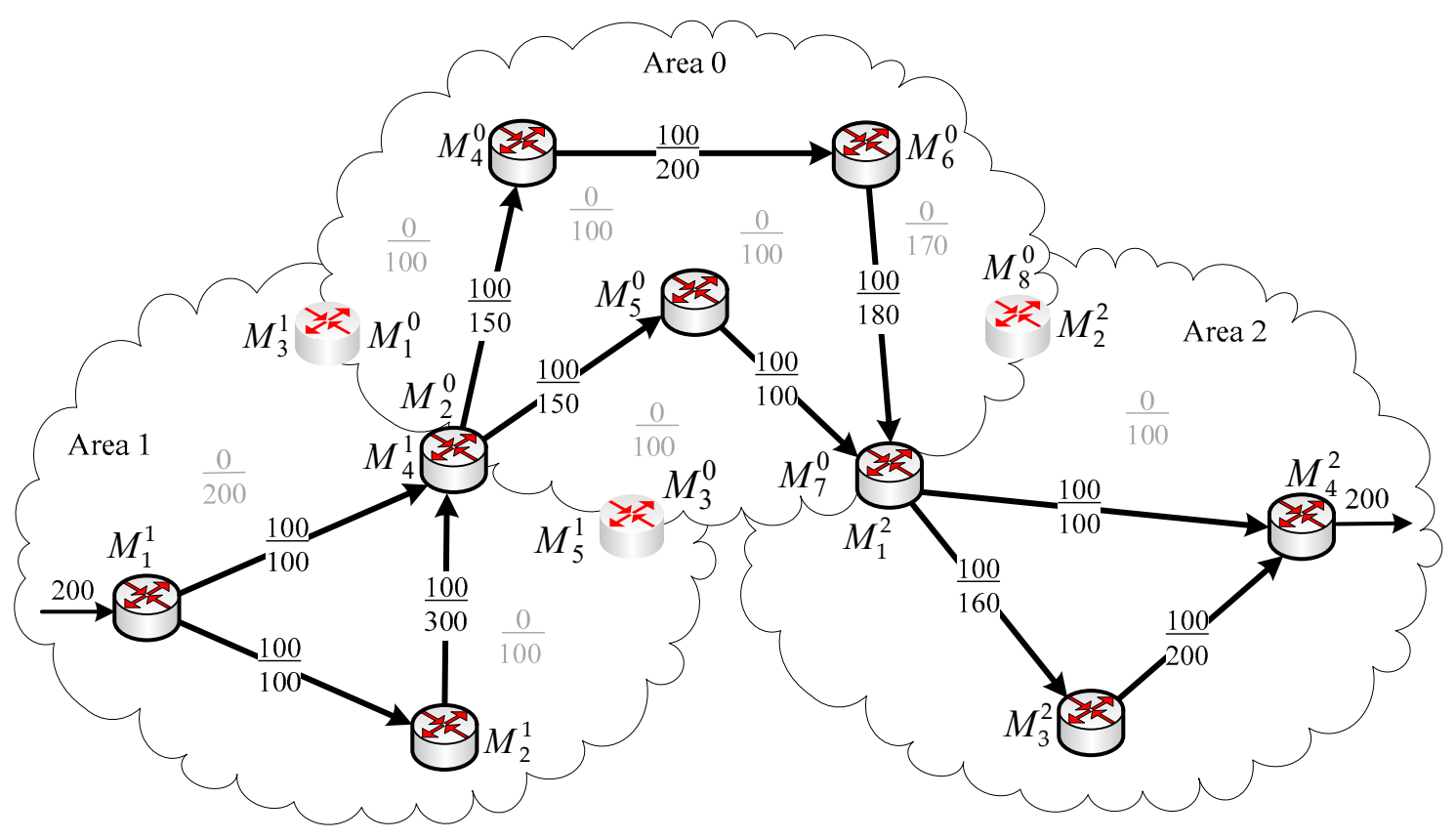

Figure 5. The resulting (coordinated) solution of the problem of inter-area multipath routing of the flow with the intensity of $2001 / \mathrm{s}$, represented by the primary multipath

Figure 5 shows the primary multipath for transmitting the flow $200 \mathrm{1} / \mathrm{s}$ from the source router $M_{1}^{1}$ to the destination router $M_{4}^{2}$, containing two paths over which flows with the same intensity of $100 \mathrm{1} / \mathrm{s}$ are transmitted:

- $M_{1}^{1} \rightarrow M_{4}^{1}\left(M_{2}^{0}\right) \rightarrow M_{4}^{0} \rightarrow M_{6}^{0} \rightarrow M_{7}^{0}\left(M_{1}^{2}\right) \rightarrow M_{4}^{2}$

- $M_{1}^{1} \rightarrow M_{2}^{1} \rightarrow M_{4}^{1}\left(M_{2}^{0}\right) \rightarrow M_{5}^{0} \rightarrow M_{7}^{0}\left(M_{1}^{2}\right) \rightarrow M_{3}^{2} \rightarrow M_{4}^{2}$.

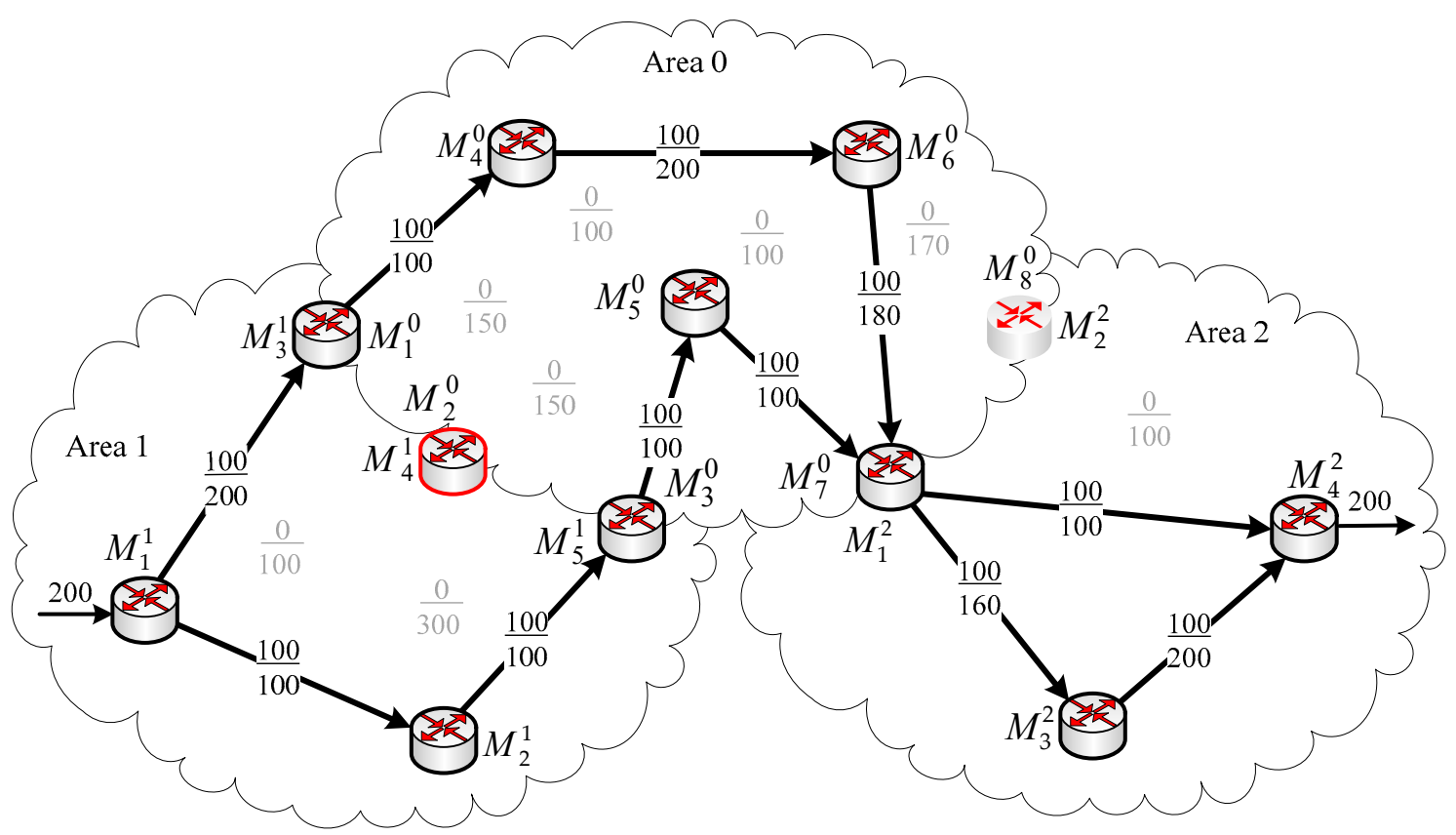

Figure 6. The resulting (coordinated) solution of the problem for inter-area multipath routing of the flow with the intensity of $2001 / \mathrm{s}$, represented by the backup multipath 
The backup multipath used to protect the border node $M_{4}^{1}\left(M_{2}^{0}\right)$ also consists of two paths (Fig. 6), over each of which the flow of the intensity $1001 / \mathrm{s}$ is transmitted:

- $M_{1}^{1} \rightarrow M_{3}^{1}\left(M_{1}^{0}\right) \rightarrow M_{4}^{0} \rightarrow M_{6}^{0} \rightarrow M_{7}^{0}\left(M_{1}^{2}\right) \rightarrow M_{4}^{2}$;

- $M_{1}^{1} \rightarrow M_{2}^{1} \rightarrow M_{5}^{1}\left(M_{3}^{0}\right) \rightarrow M_{5}^{0} \rightarrow M_{7}^{0}\left(M_{1}^{2}\right) \rightarrow M_{3}^{2} \rightarrow M_{4}^{2}$.

Given that the increase in the set of allowable solution variants in each of the areas, the solution presented in Fig. 6, has been obtained after the third iteration of the coordinating procedure (23), (24).

\section{Research of Coordination}

The effectiveness of the practical implementation of the proposed method largely depends on the speed of the coordination procedure convergence (23), (24). Minimizing the number of coordinating iterations (23) allows, firstly, to proportionally reduce the volumes of circulating service information about the state of the network; and secondly, to increase the efficiency of solving the routing problems in general. The study has found that the speed of convergence of the coordinating procedure was influenced by different factors:

- implemented routing strategy: single path or multipath;

- number of border routers;

- network loading;

- number of inter-area flows in the network;

- area and backbone structure;

- area connectivity;

- number of areas.

The presented list of factors is ordered by the degree of decrease in the influence on the speed of the procedure convergence (23), (24). This means that the number of coordinating iterations largely depended on the implemented routing strategy and the number of border routers, and to a lesser extent on the area and backbone structure, as well as the area connectivity. In general, when two border routers were used by each of the areas, the number of coordinating iterations in average was 1-2 using the single path routing or 2-3 with multipath routing (Table 2). If the area structure included three border routers, then the average number of coordinating iterations in procedure (23), (24) increased up to 2 (for single path routing) or to 4 (for multipath routing). The increase in the number of iterations was provided by the fact that with the increase in the number of border routers and the transition to multipath routing, which is sometimes caused by high network load, the number of solutions obtained in individual areas also grew, which must be coordinated with the help of the coordinating procedure (23), (24).

Table 2. The analysis results for the influence of the number of border routers and the routing strategy used on the number of iterations of the proposed method coordinating procedure (23), (24)

\begin{tabular}{|l|l|l|}
\hline \multirow{2}{*}{ Number of border routers } & Number of iterations \\
\cline { 2 - 3 } & Single path routing & Multipath routing \\
\hline 2 & 1 & $2-3$ \\
\hline 3 & $1-2$ & $3-4$ \\
\hline
\end{tabular}

During the research it was established that the size of the areas and the connectivity of intra-area routers had no significant influence on the speed of the coordinating procedure convergence.

\section{Conclusions}

In order to increase the scalability and fault-tolerance of routing solutions, the article proposes a hierarchical method of inter-area fast rerouting. The method is based on the decomposed representation of the flow-based routing model (1)-(7) with the introduction of the area interaction conditions (8) to ensure connectivity of the inter-area routes. In addition, the model includes conditions for border routers redundancy (protection), adapted for both single path and multipath routing. The proposed method of inter-area fast rerouting is based on the solution of the optimization problem associated with minimizing the congestion of the network links (20). The proposed method (21)-(24) uses the principle of goal coordination to ensure consistency of routing solutions received by the areas. In the framework of the example considered, the operability of the proposed method has been demonstrated in terms of the 
convergence of the coordination procedure (23), (24) and provision of border routers redundancy in the implementation of single or multipath routing.

The use of the proposed method allows to improve the quality of solving routing tasks in networks of hierarchical structure, namely, to significantly reduce the size of routing tables and the computational complexity of the solutions obtained by reducing the dimension of the optimization task being solved. During the research it was established that the efficiency of the proposed method in terms of the speed of the coordinating procedure convergence (23), (24) was most influenced by the number of border routers and the implemented routing strategy. In general, the method converged to optimal solutions for 1-2 iterations of the procedure (23), (24) under the single path routing, for 2-3 iterations under the multipath routing through two border routers; and for 3-4 iterations under multipath routing via three border routers.

\section{References}

1. Cisco Networking Academy (Ed.). (2014) Routing Protocols Companion Guide. Pearson Education.

2. Le Roux, J. L., Vasseur, J. P., and Boyle, J. (2005) Requirements for inter-area MPLS traffic engineering (No. RFC 4105).

3. Lemeshko, O., Nevzorova, O., and Hailan, A. (2014) The increasing convergence of coordination procedure in the implementation of multipath hierarchical routing. In: 2014 First International Scientific-Practical Conference Problems of Infocommunications Science and Technology, Kharkiv, Ukraine, October 14-17, 2014, pp.45-48. DOI: 10.1109/INFOCOMMST.2014.6992294.

4. Lemeshko, O., Nevzorova, O., and Vavenko T. (2016a) Hierarchical coordination method of interarea routing in telecommunication network. In: 2016 International Conference Radio Electronics \& Info Communications (UkrMiCo), IEEE, Kyiv, Ukraine, September 11-16, 2016, pp. 1-4. DOI: 10.1109/UkrMiCo.2016.7739626.

5. Lemeshko, O. V., Yeremenko, O. S., Tariki, N., and Hailan, A. M. (2016b) Fault-tolerance improvement for core and edge of IP network. In: 2016 XIth International Scientific and Technical Conference "Computer Sciences and Information Technologies (CSIT), IEEE, Lviv, Ukraine, September 6-10, 2016, pp. 161-164. DOI: 10.1109/STC-CSIT.2016.7589895.

6. Mesarovic, M. D., Macko, D., and Takahara, Y. (2000) Theory of hierarchical, multilevel, systems (Vol. 68). Elsevier.

7. Rak, J. (2015) Resilient routing in communication networks. Switzerland: Springer. DOI: 10.1007/978-3-319-22333-9.

8. Singh, M. G., and Titli, A. (1978) Systems: decomposition, optimisation, and control. Pergamon.

9. Tiso, J., and Teare, D. (2011) Designing Cisco Network Service Architectures (ARCH): Foundation Learning Guide. Cisco press.

10. Wójcik, R., Domżał, J., Duliński, Z., Rzym, G., Kamisiński, A., Gawłowicz, P., Jurkiewicz, P., Rząsa, J., Stankiewicz, R. and Wajda, K. (2016) A survey on methods to provide interarea multipath transmissions. Computer Networks, 108, pp. 233-259. DOI: 10.1016/j.comnet.2016.08.028.

11. Wright, B. (2003) Inter-area routing, path selection and traffic engineering. White paper, Data Connection Limited.

12. Yeremenko, O., Tariki, N., \& Hailan, A. M. (2016) Fault-tolerant IP routing flow-based model. In: 2016 13th International Conference on Modern Problems of Radio Engineering, Telecommunications and Computer Science (TCSET), IEEE, Lviv-Slavsko, Ukraine, February 23-26, 2016, pp. 655-657. DOI: 10.1109/TCSET.2016.7452143. 